\title{
A Proof of the Addition Theorem in Trigonometry.
}

I. Prove $\sin \theta=-\sin (-\theta), \cos \theta=\cos (-\theta)$.

Let $X^{\prime} O X, Y^{\prime} O Y$ be rectangular axes, and let $O P^{\prime}, O()$, starting from the position $O X$, describe angles $\theta,-\theta$. Then $O P, O Q$ are in every case symmetrically placed with respect to $O X$. (Illustrate by taking positive and negative values of $\theta$ ). Hence $x_{P}=x_{Q}, y_{P}=-y_{Q}$, and the results follow from the definitions of sine and cosine.

Cor. $\cos \theta=\cos (2 n \pi \pm \theta)$, where $n$ is any integer.

II. Prove $\cos \theta=\sin \left(\theta+\frac{\pi}{2}\right), \sin \theta=-\cos \left(\theta+\frac{\pi}{2}\right)$.

If $O P, O Q$ are radii of a circle such that $X \widehat{O O}=\theta$ and $X O Q=\frac{\pi}{2}+\theta, \mathrm{Q}$ is always a positive quadrant along the circumference from $P$. (Tllustrate by taking positive and negative numerical values of $\theta$ ). If $M, N$ are the projections of $P Q$ on $O X$, the triangles $O M P, O N Q$ are congruent, hence $x_{P}=y_{Q}$ and $y_{P}=x_{Q}$ numerically.

If $x_{p}$ is positive, $P$ is on the semi-circle $Y^{\prime} X Y$.

$$
\begin{aligned}
& \therefore Q \quad, \quad, \quad, \quad X Y X^{\prime} . \\
& \therefore y_{Q} \text { is positive. }
\end{aligned}
$$

Similarly if $x_{P}$ is negative, $y_{Q}$ is negative.

$\therefore x_{P}=y_{Q}$, and $\cos \theta=\sin \left(\frac{\pi}{2}+\theta\right)$.

Again, if $y_{P}$ is positive, $l^{\prime}$ is on the semi-circle $X Y^{\prime} X^{\prime}$.

$$
\begin{aligned}
& \therefore Q \quad, \quad " \quad Y X^{\prime} Y^{\prime \prime} . \\
& \therefore x_{Q} \text { is negative. }
\end{aligned}
$$

Similarly if $y_{P}$ is negative, $x_{Q}$ is positive.

$$
\therefore y_{P}=-a_{Q}, \text { and } \sin \theta=-\cos \left(\frac{\pi}{2}+\theta\right) \text {. }
$$

III. Prove that in any triangle $A B C, a^{2}=b^{2}+c^{2}-2 b c \cos A$. 
A PROOF OF THE ADDITION THEOREM IN TRIGONOMETRY.

IV. Prove that if $P, Q$ are the points $\left(x_{1}, y_{1}\right),\left(x_{9}, y_{2}\right)$, then $P Q^{2}=\left(x_{2}-x_{1}\right)^{2}+\left(y_{2}-y_{1}\right)^{2}$.

(Illustrate by numerical examples the fact that the projections of $P Q$ on the axes are in all cases $\left.x_{2}-x_{1}, y_{2}-y_{1}\right)$.

V. Prove that $\cos (\alpha-\beta)=\cos \alpha \cos \beta+\sin \alpha \sin \beta$, where $\alpha, \beta$ are any positive angles less than $360^{\circ}$.

If $O P, O Q$, each of length $r$, make positive angles $a, \beta$ with $O X$, the angle $P O Q$ of the triangle $P O Q$ is equal to $\alpha-\beta$ or $\beta-a$ or $360^{\circ}-(a-\beta)$ or $360^{\circ}-(\beta-a)$. (Illustrate by diagrams.)

In every case, by $\mathrm{I}, \cos P O Q=\cos (\alpha-\beta)$.

$$
\therefore P\left(Q^{2}=O P^{2}+O Q^{2}-2 O P \cdot O Q \cos (\alpha-\beta)\right.
$$

$\therefore \quad\left(x_{2}-x_{1}\right)^{2}+\left(y_{2}-y_{1}\right)^{2}=2 r^{2}-2 r^{2} \cos (\alpha-\beta)$.

But $x_{1}^{2}+y_{1}^{2}=r^{2}=x_{2}^{2}+y_{2}^{2}$.

$\therefore \quad \cos (\alpha-\beta)=\frac{x_{1} x_{2}}{r^{2}}+\frac{y_{1} y_{2}}{y^{2}}$

$$
=\cos \alpha \cos \beta+\sin \alpha \sin \beta \text {. }
$$

VI. Extend the above result to any angles.

If $A, B$ are any angles whatever, coterminal with $a, \beta$, then $A=2 m \pi+\alpha, B=2 n \pi+\beta$, where $m$ and $n$ are integers, positive or negative.

$\therefore \quad A-\not B=2 k \pi+(\alpha-\beta)$, where $k$ is an integer.

$\therefore \quad \cos (A-B)=\cos (\alpha-\beta)$

$$
\begin{aligned}
& =\cos a \cos \beta+\sin \alpha \sin \beta \\
& =\cos A \cos B+\sin A \sin B \text { (by I, Cor.). }
\end{aligned}
$$

VII. By means of I and II extend the result to $\cos (A+B)$, $\sin (A-B), \sin (A+B)$ in the usual way.

R. J. T. BeLL. 